\title{
Ciudades y urbanismo en el mundo antiguo: una introducción
}

\author{
(1) Pablo Jaruf \\ Universidad de Buenos Aires / Universidad Nacional de Luján / Instituto Superior del \\ Profesorado “Joaquín V. González”, Argentina
}

Fecha de recepción: 25 de julio de 2021. Fecha de aceptación: 18 de agosto de 2021.

\section{Resumen}

El objetivo de este artículo es introducir de manera breve los debates en torno al concepto de ciudad y de urbanismo, con especial referencia al mundo antiguo. Con respecto a la ciudad, se sintetizan los aportes realizados por las ciencias sociales desde mediados del siglo XIX en adelante, resaltando aquellos estudios comparativos que han pretendido identificar sus rasgos comunes en distintas regiones y períodos. También se presta atención a aquellos enfoques que enfatizan las nociones nativas del pasado sobre aquello que nosotros hoy consideramos como ciudades. A continuación, se profundiza en los aportes del urbanismo, a partir del cual se comienza a estudiarlas como nodos de un sistema jerárquico de asentamientos, predominando entonces las relaciones y oposiciones con respecto a otras formas de ocupación del territorio, como aldeas rurales o tiendas de nómades. Por último, antes de presentar las contribuciones que forman parte de este dossier, se propone una definición de ciudad.

Palabras clave: ciudad, urbanismo, mundo antiguo, ciencias sociales

\section{Cities and Urbanism in the Ancient World: An Introduction}

\begin{abstract}
The aim of this paper is to briefly introduce the debates about the concepts of city and urbanism, with special attention to the ancient world. Regarding the city, the contributions made by the social sciences from the middle of the 19th century onwards are summarized, highlighting those comparative studies that have tried to identify the common features of cities in different regions and periods. Attention is also paid to those approaches that emphasize native
\end{abstract}


notions about what we today regard as cities. Then, it delves into the contributions of urbanism, studying cities as nodes of hierarchical settlement systems, prevailing relations, and oppositions, with respect to other forms of landscape occupation such as rural villages or nomad tents. Lastly, before introducing the contributions that are part of this dossier, a definition of the city is proposed.

Keywords: city, urbanism, ancient world, social sciences

\section{Las ciudades antiguas y el problema de las definiciones}

"City", "urban site", "urban society", and "urbanization" are often
undertheorized, and it is easy to find publications that leave these
terms undefined and assume that we all know what they mean.

Cowgill (2004: 526)

La ciudad en el mundo antiguo es un tópico muy concurrido a la hora de la investigación. Esto se debe a que la mayor parte de la evidencia, tanto textual como arqueológica, procede de asentamientos urbanos. A su vez, es común asociar los grandes cambios sociales con la emergencia o la transformación de las ciudades, como por ejemplo el surgimiento de las primeras urbes en Mesopotamia o la modificación del paisaje urbano en el mundo egeo con la formación de las póleis. Si nos adelantamos a la Edad Media, también es común considerar a los pequeños burgos como un agente de cambio central en el origen del capitalismo comercial y, por ende, del mundo moderno.

En general, predominan dos enfoques: aquel que se dedica a establecer las continuidades entre las ciudades antiguas y modernas, considerando a las primeras como la base del urbanismo actual; y aquel otro que, al contrario, destaca los cambios y las rupturas, planteando que las ciudades previas a la industrialización poco o nada tienen que ver con las del mundo contemporáneo. Un aspecto que complejiza este tipo de comparaciones es el carácter variable de la ciudad moderna, pues si bien en un principio se caracterizaba por la industria, luego se transformó en un centro financiero y de servicios, convirtiéndose hoy en una megaciudad, una cosmópolis -por su interconexión global-, e incluso en una suerte de postmetrópolis, es decir, en un espacio dinámico de cambio continuo en el que ya no es posible establecer con seguridad sus rasgos definitorios (por ej., Soja, 2008 [2000]).

Las ciudades antiguas, de esta manera, son incorporadas como parte de un grupo mayor, las ciudades preindustriales, las cuales suelen ser comparadas entre sí para identificar sus rasgos comunes o, al contrario, sus diferencias (por ej., Sjoberg, 1960; Storey, 2006; Farhat, 2020). En un principio, aquellas que se ubicaban cerca o a orillas del Mediterráneo durante la época clásica, como por ejemplo Atenas o Roma, eran consideradas como las ciudades por antonomasia, ocupando entonces un lugar central en dichas indagaciones. Es lógico que esto fuera así pues la propia palabra procede del latín civitas, lo cual implicaba sobre todo una serie de instituciones basadas en la ciudadanía. Dicho rasgo, asociado al tipo de estructura política, ha dado lugar a estudios célebres, como el de Fustel de Coulanges (2009 [1866]), de mediados del siglo XIX, cuyo 
análisis combinaba la tradición filológica con los aportes de la, por entonces, naciente disciplina sociológica. Aquellos pocos que entonces se animaban a incluir ciudades de otras latitudes lo hacían para destacar la libertad política europea, como la famosa comparación entre la ciudad occidental y la oriental realizada a comienzos del siglo XX por Max Weber (1944 [1922]: 938-1024).

Claro que trabajos como el antedicho se basaban casi exclusivamente en fuentes europeas y evidenciaban un desconocimiento profundo sobre Oriente, así como de otras partes del mundo, entendible entonces por el escaso desarrollo de disciplinas tales como la arqueología y la antropología. En efecto, una vez que comenzó a aumentar la información y se modificó el punto de vista de los investigadores, las ciudades antiguas de los demás continentes pasaron a convertirse en la piedra angular de los estudios sobre los grandes cambios de la historia humana. El aporte más significativo fue realizado por el arqueólogo Gordon Childe (1950) quien, basado en el modelo de la revolución industrial, consideró a las primeras ciudades de Mesopotamia, de Egipto, del valle del Indo y de Mesoamérica, como centros donde ya predominaba una solidaridad orgánica, es decir, una relación interdependiente entre personas que desempeñaban distintas tareas, cuya coordinación dependía de un grupo gobernante que se imponía con el fin de evitar conflictos derivados de las nuevas formas de desigualdad que estas dinámicas implicaban. Las evidencias materiales de este fenómeno no eran sólo el aumento del tamaño de los asentamientos y su arquitectura monumental, sino también los artefactos cuya elaboración denotaba un alto grado de técnica, seguramente resultado de un trabajo artesanal especializado, además del surgimiento de formas de registro, como la escritura, y el desarrollo de ciencias exactas, entre otros indicadores.

A partir de entonces ha sido común hablar de ciudades en Mesoamérica y los Andes precolombinos, el África subsahariana, el subcontinente de la India, Asia Central, Asia Oriental, el Sudeste asiático, e incluso Europa antes de la época clásica (por ej., Smith, 2003; Marcus y Sabloff, 2008; Clark, 2013; Yoffee, 2015; Frangipane y Manzanilla, 2018). La comparación entre esta enorme variedad de casos ha permitido detectar notables diferencias, lo que en ocasiones ha derivado en discusiones dedicadas a distinguir las características primarias de las secundarias (por ej., Redman, 1990 [1978]: 281-283). Un tema de debate ha sido el tamaño mínimo para definir una ciudad pues, por ejemplo, ¿qué cosas habría en común entre asentamientos que contaban con más de 700 ha, como Yinxu, de fines de la Dinastía Shang (ca. 1300-1500 a.C.), y otros que no superaban las 50 ha, como Argos, en el mundo egeo durante la Edad de Hierro (ca. 1050-750 a.C.)? Sin embargo, conviene recordar que el núcleo de la definición de Childe era funcionalista y que, si bien contemplaba la cuestión del tamaño, éste no era al aspecto principal, como varios investigadores se han encargado de señalar (por ej., Trigger, 1972; Redman, 1990 [1978]: 281-288; Lull y Micó, 2007: 192-202; Liverani, 2014 [2013]: 151-158).

Otros debates se basaron más bien en la representación ideal de la ciudad. Desde este punto de vista, no alcanzaba con inferir, a partir de los restos materiales, la existencia de grupos de especialistas, de gobernantes y de desigualdades sociales, sino que era necesario también identificar un conjunto de ideas que le otorgaran a este tipo de asentamiento una función o un valor superlativo con 
relación a otras formas de ocupación del territorio. Esta cuestión no era fácil de demostrar, pues muchas sociedades antiguas eran ágrafas, mientras que en aquellas donde existía escritura no siempre establecían distinciones claras. Por ejemplo, si bien en los textos cuneiformes de la Mesopotamia encontramos la palabra acadia ālum o el sumerograma URU, muchas veces es empleado también para referirse a pequeños poblados rurales que en nada se asemejan a lo que entendemos por ciudad (por ej., van de Mieroop, 1997: 9-11). En el caso de los documentos de Ebla, en Siria antigua, se complica aún más, pues allí se utilizaba el sumerograma KI, con lo cual se referían a la capital, a secciones dentro de la misma, a pequeñas aldeas e incluso a pozos de agua ubicados en el desierto (Biga, 2013). Una situación parecida ocurre con los documentos jeroglíficos egipcios, donde para referirse a distintos tipos de asentamiento se utilizaba un mismo término, niut (por ej., Parlebas, 1983).

De todas formas, un estudio más detenido de los documentos, así como también el propio desarrollo histórico de las distintas formas y funciones de los asentamientos, permiten entrever la emergencia de formas de conciencia donde existen lugares centrales y se reconoce una jerarquía espacial. Para citar nuevamente los documentos cuneiformes, podemos mencionar el vocablo acadio de kaprum, que se utilizaba para referirse a un asentamiento de carácter rural, lo que perdura luego en el uso de la raíz semítica $k p r$ tanto en las tablillas de Ugarit como en la Biblia Hebrea (por ej., Milevski, 2010: 165-167). A lo anterior hay que sumar, además, la temprana formación de una conciencia citadina que se contraponía claramente al mundo rural, lo cual es evidente ya en los textos literarios sumerios (por ej., Verderame, 2010).

Más allá de las barreras lingüísticas, los estudios sobre el simbolismo urbano lograron un gran desarrollo -sobre todo a partir de la publicación del libro de Paul Wheatley dedicada a las ciudades chinas (1971)- cuando consideraron la planificación interna y sus monumentos como la creación de un verdadero microcosmos. Esto, claro está, es más evidente en sitios de nueva fundación, construidos muchas veces tras el ascenso de un nuevo monarca, como sucedía en el Sudeste asiático, Egipto o Mesoamérica (por ej., Carl et al., 2000). Para el Cercano Oriente este tipo de estudios parecía menos útil, pero trabajos sobre ciudades de Siria y de Anatolia han demostrado su ventaja, pues aún en asentamientos muy pequeños existían nociones compartidas sobre el uso del espacio urbano (por ej., Harmanşah, 2013; Osborne, 2014).

Hoy en día es común distinguir, por un lado, entre aquellas ciudades que parecen haberse formado por una aglomeración imprevista, denominadas en inglés como primate o pristine -u orgánicas- en el sentido que surgen a partir del crecimiento de asentamientos antes rurales, y, por otro lado, las ciudades disembedded o planted -o fundaciones-, es decir, aquellas que fueron construidas en suelo virgen, siguiendo una estricta planificación. ${ }^{1}$ Para citar un ejemplo temprano, contamos con el sitio de Habuba Kabira-Tell Kannas, de fines del período Calcolítico,

1 Para profundizar en estas conceptualizaciones, véase Cowgill (2004: 543-537) y Diego Espinel (2002: 19-22). Para la diferencia entre una simple aglomeración y una ciudad propiamente dicha, véase el artículo de Mizzoni en este volumen. 
ubicado en el Éufrates medio, donde es evidente el empleo de técnicas y de modelos ya conocidos en el sur de la Mesopotamia (por ej., Finet, 1982). Dicho esto, en realidad lo más probable es que ambos tipos de ciudades coexistan en un mismo asentamiento: aquellos que incrementan su tamaño pueden remodelar las partes centrales, construyendo complejos de edificios públicos, lo que para Roma es tomado, por ejemplo, como el paso de una simple aglomeración a una verdadera ciudad (López Barja de Quiroga y Lomas Salmonte, 2004: 48-49); a su vez, estas urbes pueden crecer de manera desmedida décadas o siglos después, rodeándose de barrios periféricos que en principio carecen de una planificación, obligando a reformas ulteriores (por ej., Mandich, 2019).

Sucede que las ciudades, como todo fenómeno histórico, cambian, se modifican, por lo que aquello que en un contexto era entendido como una ciudad, puede ya no serlo en otro. Este carácter dinámico complica las comparaciones, ya que un mismo asentamiento puede variar lo suficiente hasta perder aquellos rasgos que en una primera impresión asociábamos con una ciudad. Establecer diferencias y semejanzas es una operación básica que, en el caso de las ciudades, nos permite discernir sus rasgos característicos, pero hay que advertir que en ocasiones nos puede hacer caer en razonamientos circulares, pues ¿cuál ha sido el criterio que se ha utilizado para seleccionar los asentamientos a partir de los cuales se infieren los rasgos comunes de las ciudades? ¿Acaso, en esa selección, no se está asumiendo ya un concepto de ciudad?

Una forma de justificar esta especie de tautología es que dicho recorrido al menos permite otorgar fundamento teórico a lo que en un principio eran sólo nociones imprecisas. Algunos, tratando de sortear estas trampas, han decidido incluir otro tipo de asentamientos que, si bien no suelen ser considerados como ciudades, poseen algunos rasgos de éstas, para llegar así a conceptos más abarcadores. Un ejemplo de lo anterior es la siguiente definición que ofrecen Norman Yoffee y Nicola Terrenato en el tercer volumen de la colección Nueva Historia de Cambridge, dedicado de forma integral a las ciudades antiguas (2015: 1-2):

...las ciudades son asentamientos permanentes de mayor tamaño y población, varios miles de habitantes, que residen muy cerca unos de otros y que son socialmente diversos; existen líderes y sus subalternos, que controlan el seguimiento de las personas y cosas en la ciudad, lo que entra y sale de ella; las ciudades tienen un centro con arquitectura imponente que permite $y / o$ restringe la actividad política, social y/o ideológica; las ciudades dependen de los alimentos que se producen en las campiñas aledañas; las ciudades proveen determinados servicios y productos manufacturados a la población de las campiñas aledañas y adquieren, a través del comercio a larga distancia, bienes de lujo y utilitarios; las ciudades proporcionan un sentido de identidad cívica a las personas que viven en ella ( $y$ a las zonas aledañas), y a su vez son arenas en donde los gobernantes demuestran sus conexiones especiales con los grandes dioses y el cosmos; y las ciudades son contenedoras del potencial descontento y drama social entre varios grupos sociales que compiten/cooperan y sus líderes locales; las ciudades crean e incuban problemas ambientales y de salud significativos. ${ }^{2}$

2 Traducción propia del inglés al castellano. 
La ventaja de esta última definición es que no sólo destaca la función que cumple la ciudad sino también las dinámicas que se generan en su interior y zonas aledañas, prestando especial atención a los conflictos y los problemas que generan dichas aglomeraciones.

Sin embargo, esta suerte de lista, pero dispuesta a modo de prosa, presenta el mismo problema recurrente: ¿cuántos indicadores hacen falta para identificar a una ciudad? La mejor manera de solucionar este dilema, a nuestro modo de ver, no residiría en seguir sumando características, sino en sintetizar una o dos ideas centrales que permitan comprender, justamente, aquellos rasgos distintivos que son propios de la vida urbana. Tanto Vicente Lull y Rafael Micó, como Mario Liverani, basándose en la propuesta de Childe, han ofrecido definiciones en este sentido. Los primeros sostienen que la ciudad se basa en la "concentración y gestión centralizada de excedentes producidos socialmente" y la "división del trabajo que contempla la especialización a tiempo completo entre quienes producen y quienes gestionan el excedente social acumulado" (2007: 200). Por su parte, Liverani plantea que "la ciudad se distingue de la aldea por una complejidad que se basa en la especialización laboral y produce desigualdades socioeconómicas" y en "una separación de excedentes (motivada ideológicamente), que se centralizan y redistribuyen a los productores" (2014 [2013]: 154). Obsérvese que estas definiciones no hacen referencias explícitas a los rasgos que solemos asociar más comúnmente con las ciudades, como el tamaño o la arquitectura monumental, sino que esto último sería la expresión tangible de la existencia de un sistema social basado en la división del trabajo especializado y la gestión centralizada del excedente. ${ }^{3}$

Claro que en este tipo de propuestas predomina el peso de lo redistributivo, centralidad puesta en duda por otros enfoques que prefieren destacar las prácticas de intercambio semejantes al comercio. ${ }^{4}$ Según estos enfoques, la aglomeración tiene como objetivo maximizar los beneficios, concentrándose las poblaciones en lugares donde es más accesible la participación de rutas de intercambio o el control de determinados recursos (por ej., Hirth, 1978; Silver, 2004). En todo caso, la emergencia de instituciones políticas sería el resultado del crecimiento de este tipo de asentamientos basados en el intercambio, ya sea por acuerdo mutuo o la imposición violenta de un grupo local o de conquistadores extranjeros (por ej. Marfoe, 1979; Stone, 2005).

Llegados a este punto, entonces, es evidente que para comprender y definir qué es una ciudad, no alcanza con determinar sus rasgos característicos, sino que es necesario considerar todo aquello que está por fuera de la misma, ya sea la campiña rural donde se obtienen los excedentes que son concentrados y redistribuidos, o aquellos otros lugares y personas que conectan las rutas de

3 Sobre la centralidad de la división del trabajo especializado al interior del espacio urbano, véanse los trabajos de Rovira y de Getzov y Milevski en este número.

4 Esta contraposición entre redistribución y comercio retoma el debate secular entre primitivistas y modernistas, que en el caso de las ciudades se replica en la oposición entre ciudad consumidora y ciudad mercantil (por ej., Fox, 1977). 
intercambio. En efecto, la ciudad es sólo una parte de un sistema mayor, sin el cual su propia existencia sería imposible.

\section{Ampliando la escala: las ciudades como nodos de sistemas urbanos}

A partir de la década de los sesenta, tanto desde el campo de la arqueología como de la antropología, ha sido común aplicar modelos y conceptos generados por la geografía y la disciplina urbanística, principalmente la teoría de los lugares centrales, propuesta por Walter Christaller (1980 [1933]) y ampliada por investigadores posteriores (por ej., Berry y Pred, 1961). Según esta teoría, existe una jerarquía de asentamientos dispuestos de forma tal que permite la circulación más eficiente de personas, de bienes y de ideas. Tanto el número como el tamaño y la ubicación de éstos depende de los costes de transporte, el acceso a la tierra agrícola, la escala de la producción, entre otros factores.

Un estudio pionero en aplicar estos enfoques a las ciudades antiguas fue el de Robert McC. Adams, quien realizó prospecciones en la Baja Mesopotamia (por ej., 1965), cuyos resultados fueron comparados con los sistemas de asentamiento de Mesoamérica (1966). A esto se sumó el trabajo de Gregory Johnson (1972) en Irán sudoccidental, donde estudió la urbanización temprana de la región de Elam. Rápidamente, este tipo de abordaje fue replicado en distintas partes del mundo, convirtiéndose en un elemento característico de la Nueva Arqueología o Arqueología Procesual (por ej., Hodder y Orton, 1990 [1976]). Incluso, debates tales como el origen del Estado pasaron a concentrarse en los cambios en los sistemas de asentamiento, donde para algunos una jerarquía de dos o tres niveles equivalía a una jefatura, mientras que una de tres o cuatro ya correspondía a una sociedad estatal (por ej., Wright y Johnson, 1975; Service, 1984 [1975]: 93-98; Flannery, 1998).

Este tipo de estudios siempre estuvo caracterizado por un peso importante de las matemáticas y, por lo tanto, de los aspectos cuantitativos, pero los últimos años esto se ha matizado bastante, debido a la aplicación de la fenomenología del paisaje, gracias a lo cual ganaron mayor presencia los aspectos cualitativos, es decir, la percepción subjetiva que tienen las personas sobre el espacio que habitan (por ej., Tilley, 1997). La relación entre los asentamientos y la ubicación de estos ya no responde sólo a maximizar beneficios sino también, por ejemplo, a la identificación con un paisaje sacralizado, a la mayor o menor visibilidad, así como a la configuración de mapas mentales que en ocasiones replican el movimiento de los astros, entre otros aspectos (por ej., Ashmore, 2008; van Dike, 2008).

Más allá de las diferencias, lo que tienen en común todos estos enfoques es que las ciudades siempre son consideradas como parte de sistemas regionales. En general, podemos remitirnos al trabajo clásico de Charles Redman (1990 [1978]: 278-279), quien entendía a la ciudad "como un nódulo de funcionamiento integrado en una red más extensa" que "tan sólo puede ser evaluado en términos del sistema en su conjunto", definiéndose entonces "como una intersección de la red". 
El aspecto distintivo de estos enfoques es que incorporan dentro de los estudios urbanos a los poblados y aldeas rurales. Como sostiene George Cowgill (2004: 527), "lo rural sólo tiene sentido como un sector dentro de sociedades que también tienen un sector urbano"; "todos los asentamientos tienen áreas de captación (catchment areas), pero sólo las ciudades tienen campiñas (hinterlands)". Incluso se ha vuelto común analizar la relación entre nómades y sedentarios como partes interdependientes de un mismo amplio sistema (por ej., Khazanov, 1984 [1983]), llegando algunos a sugerir que la especialización a tiempo completo en el pastoralismo sería resultado de una vinculación sistémica con los centros urbanos ${ }^{5}$ (por ej., Rosen, 2017: 167-204).

De esta manera, hoy podemos encontrar definiciones que ya no se centran tanto en las características intrínsecas de las ciudades, sino en su oposición a otras formas de asentamiento o modos de vida móviles. Por ejemplo, en arqueología se cuenta con la propuesta de Cowgill, para quien una ciudad es:

un asentamiento permanente en un territorio más amplio ocupado por una sociedad considerada hogar por un número significativo de residentes cuyas actividades, roles, prácticas, experiencias, identidades y actitudes difieren considerablemente de aquellos miembros de la sociedad que se identifican mayormente con las tierras 'rurales' fuera de tales asentamientos (2004: 526-527). ${ }^{6}$

Por su parte, Jürgen Osterhammel, desde el enfoque de la historia global, sostiene que la ciudad es "una forma de organizar socialmente el espacio" que "siempre se halla en tensión con alguna otra cosa, la 'no-ciudad", que "puede ser muy variada: el 'campo' con sus pueblos y aldeas de campesinos estables; los desiertos y las estepas de los nómadas; el mundo de las grandes haciendas y plantaciones, donde se concentra el poder de los terratenientes" (2015 [2013]: 351).

Es evidente el peso que en este tipo de definiciones ha ganado la disciplina urbanística, la cual se creó a fines del siglo XIX para comenzar a analizar el crecimiento, organización y planificación de las ciudades modernas. ${ }^{7} \mathrm{La}$ utilidad de sus enfoques aplicados al mundo antiguo ha motivado que arquitectos, urbanistas y sociólogos se animaran a realizar síntesis que partían de los primeros asentamientos prehistóricos hasta llegar a las grandes urbes contemporáneas (por ej., Mumford, 2012 [1961]). En este tipo de síntesis predominan las continuidades, dejando de lado esa división tajante entre ciudades preindustriales e industriales, o entre antiguas y modernas, sosteniendo que los mismos conceptos pueden servir para analizar tanto el presente como el pasado. Un arqueólogo que ha seguido fielmente esta orientación es Guillermo Algaze, quien ha estudiado el surgimiento de las ciudades mesopotámicas a partir de los mismos modelos propuestos, por ejemplo, para Chicago o Nueva

5 Sobre la relación entre los pastores y los centros urbanos, véanse los artículos de Cismondi y de Kifer en este número.

6 Traducción propia del inglés al castellano.

7 Para un compilado de reseñas en español sobre libros clásicos de urbanismo moderno, véase Di Biagi (2014). 
York (2008: 17-19, 29-38, 43-53), e incluso ha sugerido que debieron existir problemas de salud semejantes a los que se generaban en las urbes europeas de la modernidad temprana, como Londres durante los siglos XVI-XVIII (2018).

Una consecuencia evidente de estos enfoques sistémicos es que la ciudad pierde sus rasgos propios para pasar a definirse por el tipo de relaciones que establece con otros asentamientos. Desde este punto de vista, no importa el tamaño, la arquitectura, la planificación, en pocas palabras, el aspecto físico de una ciudad, en tanto y en cuanto desempeñe la función central en un sistema jerárquico de asentamientos. Por ejemplo, Egipto, que solía ser pensado como una "civilización sin ciudades" (Wilson, 1960), ahora es cada vez más común que sea considerado como plenamente urbano pues, más allá de la cantidad y el tamaño de sus ciudades, existían múltiples centros que desempeñaban aquellas funciones que hoy caracterizamos como urbanas, especialmente los grandes complejos funerarios, focos de concentración de trabajadores dedicados a labores especializadas (por ej., Gloria Estúa, 2003; Bietak et al., 2010; Moeller, 2016). De la misma manera, la región de Angkor, ocupada por centros ceremoniales de baja densidad demográfica, no solía ser considerada como urbana (por ej., Coe, 1961), apreciación que luego comenzó a cambiar debido, por un lado, a la enorme cantidad de personas que debieron trabajar en su construcción y que luego peregrinaban, y, por otro lado, a la constatación de las distintas formas de asentamiento altamente integradas que se encontraban dispersas en toda esta región (por ej., Evans et al., 2007; Carter et al., 2018).

Pero la principal consecuencia de estos estudios es que, por más pequeño que sea un sistema de asentamiento, casi siempre es posible inferir cierta jerarquía. Por esta razón, varios investigadores sostienen que el urbanismo como tal surge ya en la prehistoria, mucho antes de la aparición de ciudades como Uruk en Mesopotamia. ${ }^{8}$ Alexander Thomas (2012), por ejemplo, habla de "urbanización antes de las ciudades", planteando que desde la cultura neolítica Halaf es posible identificar vínculos asimétricos entre asentamientos. Algo semejante proponen Garfinkel y Ben-Shlomo (2009) para otra cultura neolítica, la Yarmukiense, donde en sitios como Shàar Hagolan advierten indicios de planificación urbana. Pero los debates llegan tan atrás como el Neolítico Precerámico (por ej., Verhoeven, 2006), por lo que, de esta manera, recuperan actualidad aquellas teorías que consideraban a Jericó como la primera ciudad, como insiste Edward Soja (2008 [2000]: 62-72), e incluso resurgen conjeturas propuestas en su momento por Jane Jacobs (1975 [1969]: 9-57), quien sostenía que las primeras ciudades habían sido construidas por cazadores-recolectores.

Claro que una forma de aportar más rigurosidad es reservar el adjetivo urbano, como dijimos antes, para esos sistemas que cuentan con una jerarquía de tres niveles como mínimo. Aunque esta cuestión nos lleva al problema sobre cómo identificar distintos niveles al interior de un sistema. Un criterio recurrente ha sido el tamaño, por ejemplo, distinguiendo entre asentamientos pequeños, medianos y grandes, pero es evidente que este tipo de clasificación depende de

8 Sobre evidencias tempranas de urbanismo en el Cercano Oriente, véase el artículo de Leandro Constanze Lima en este volumen. 
la arbitrariedad del investigador, problemática que ya había señalado Richard Blanton (1976). Sin embargo, no siempre los asentamientos más grandes desempeñan una función central, advertencia realizada por Liverani (2014 [2013]: 277-279), quien resalta la relevancia de la descentralización de funciones en los sistemas urbanos de la antigua Mesopotamia. ${ }^{9}$

Por supuesto, el mejor antídoto sería combinar la evidencia arqueológica con la epigráfica, lo que permitiría definir más claramente la función desempeñada por los distintos asentamientos, aún con las barreras lingüísticas comentadas en párrafos anteriores. Otro aspecto que ayudaría a precisar es considerar también la antedicha representación ideal de ciudad, es decir, identificar aquellos principios sobre planificación y arquitectura que son propios de los asentamientos urbanos en un contexto regional e histórico específico.

En suma, se torna evidente que para lograr una definición más precisa de ciudad no sólo es necesario dar cuenta de las relaciones y oposiciones con otro tipo de asentamientos, sino también considerar los elementos característicos que las distinguen de estos últimos. Sin ánimo de agotar el debate, nos parece entonces que una manera de abordar el estudio de las ciudades podría ser considerarlas como aquellos centros habitados por poblaciones diversas que ocupan, ocuparon, o se espera que ocupen, la cima (o los niveles más altos) de un sistema jerárquico de asentamiento -caracterizado éste por una división espacial del trabajo y la centralización de excedentes-, distinguiéndose por una planificación y una arquitectura propia, según dicten los criterios culturales predominantes de un período $y$ de una región en particular.

El empleo de tres tiempos verbales permite incorporar el rasgo histórico de las ciudades, su permanente cambio, pues bien puede ser que un asentamiento haya cumplido su función en un momento determinado, pero luego ya no, o que, al contrario, se construya todo un nuevo asentamiento esperando que se convierta en el centro de un sistema regional, como el caso de las antedichas ciudades disembedded o planted. Con relación a esto último, es conveniente señalar que éstas se caracterizan también por servir como espacio de ensayo, es decir, donde se llevan a la práctica nuevas ideas sobre planificación y arquitectura, convirtiéndolas en visiones del futuro. ${ }^{10}$ Es evidente también que hay determinadas ciudades cuyo diseño y arquitectura ya no responden a los criterios de una época, pero eso no le quita su centralidad, que viene dada por el prestigio pasado, ni que decir que las grandes urbes suelen contener en su fisonomía elementos de distintas épocas, convirtiéndolas en verdaderos "archivos de la historia" (Chueca Goitia, 1968: 24-43).

9 Sobre la dificultad de considerar el tamaño como criterio para determinar la jerarquía de un sistema de asentamiento, véase el artículo de Villagrán y Fini en este número. Para un estudio sobre la relación jerárquica entre distintos asentamientos en un caso específico sobre una ciudad mesopotámica, véase el artículo de Bramanti en este número.

10 Sobre la ciudad como espacio de utopía que motiva programas de reforma urbana podemos citar, para el mundo antiguo, La república (ca. 370 a.C.) de Platón, y, para el mundo moderno, Garden Cities of Tomorrow (1902) de Ebenezer Howard. 
Con respecto a la división espacial del trabajo y la centralización del excedente, se trataría de los aspectos que definen, por un lado, la interdependencia y, por el otro, la jerarquización del sistema, así como la cantidad de niveles de éste. La centralización del excedente puede y suele darse a través de distintas vías, ya sea por la imposición de un tributo o bien por la existencia de plazas o puertos de mercado donde los actores acuden para intercambiar sus productos. Una mengua en la división del trabajo, donde en distintos asentamientos se comiencen a producir los mismos bienes, así como una mayor descentralización de excedentes en el espacio rural, llevan como resultado la desurbanización. Por ejemplo, estos factores, sumados a la pérdida de territorios, son los que explican para muchos la decadencia de la mitad occidental del Imperio romano, a diferencia de su mitad oriental, que todavía gozaba de una próspera vida urbana (por ej., Wickham, 2013 [2009]: 74-82).

Por supuesto, cuanta mayor evidencia se disponga y mejor se puedan precisar los distintos aspectos de un asentamiento, más seguridad se tendrá para afirmar si es urbano o no. Pero, si, al contrario, el registro es escaso y la información ambigua, el carácter urbano permanecerá sólo como hipótesis de trabajo, como orientación para profundizar en aquella cuestión que uno se proponga investigar. Va de suyo que una definición como la propuesta puede que no resuelva todo lo que implica el fenómeno urbano, pero al menos sirve como base para luego abordar aspectos más específicos. Como cualquier modelo o concepto, a medida que aumenten los conocimientos y nuestro propio mundo se transforme, es preciso revisarlo y redefinirlo para así lograr una mejor comprensión, tanto de la realidad presente como de la pasada.

\section{Presentación del dossier}

Desde mediados de 2019 hasta fines de 2021, el equipo de investigación del proyecto FiloCyT 19-075 titulado "Ciclos de urbanización y dinámicas sociopolíticas en Siria-Palestina: de la Edad del Bronce a la Edad del Hierro (3600-700 a.C.)", perteneciente a la Facultad de Filosofía y Letras de la Universidad de Buenos Aires (UBA), y radicado en el Instituto de Historia Antigua Oriental (IHAO), se propuso indagar en las características del urbanismo antiguo, pero trabajando de manera específica los problemas relativos a los ciclos de urbanización y el desarrollo de las ciudades en Siria-Palestina durante las Edades del Bronce y del Hierro, focalizándose en los cambios sociopolíticos que dichos procesos ponían de manifiesto.

Este proyecto estuvo integrado por doctores (Pablo Jaruf y Augusto Gayubas), licenciados (M. Belén Daizo), profesores (Alejandro Mizzoni) y estudiantes (Melina Fini, Leandro Constanze Lima y Katherine Kifer), no sólo de la carrera de Historia sino también de Antropología con orientación arqueológica, contando también con la colaboración externa de especialistas de otras universidades (Dra. Leticia Rovira, Universidad Nacional de Rosario y Universidad Nacional del Litoral; y Dr. Bernardo Gandulla, docente jubilado de la UBA y de la Universidad Nacional de Luján). 
A lo largo de estos últimos dos años nos reunimos, tanto de manera presencial como virtual, para debatir los distintos aspectos, tanto del proyecto común como de las investigaciones personales que cada uno llevaba adelante. Este intercambio se vio enriquecido con la invitación de otros investigadores, quienes participaron de un ciclo de entrevistas, las cuales se encuentran disponibles en un canal de YouTube: Instituto de Historia Antigua Oriental [FFyL-UBA]. Dicha iniciativa sirvió como puntapié para avanzar en la realización de una publicación conjunta, donde quedaran plasmados tanto nuestros avances como el diálogo con los colegas, cuyo resultado es el dossier que aquí se presenta.

Ante todo, nos parece importante resaltar la incorporación de espacios históricos que no suelen ser considerados en la revista de nuestro instituto, pero que ahora tienen lugar con motivo del tema común que nos reúne: las ciudades y el urbanismo en el mundo antiguo. Estamos haciendo referencia a los artículos de Linda Manzanilla y de Ignacio Villagrán y Melina Fini, quienes trabajan Mesoamérica y Asia oriental respectivamente. El primero, "Ciudades emblemáticas del pasado: de la ciudad primigenia (Uruk-Warka) a la megalópolis arquetípica (Teotihuacan)", parte de Sumeria para pasar rápidamente a un análisis detenido de la gran ciudad del período clásico mesoamericano. Además de destacar el carácter multiétnico de las poblaciones que habitaban los distintos barrios de Teotihuacan, sugiere que en la configuración del asentamiento puede advertirse una competencia entre dos modelos de organización social, uno más excluyente y otro más corporativo, lo que nos remite a esa idea de pensar la ciudad como arena de exhibición y de drama social que planteaban Yoffee y Terrenato (ver más arriba). El otro artículo, "Redes de ciudades en China imperial temprana (siglos III a.e.c.-III e.c.). Control territorial y lógicas de parentesco", también nos permite adentrarnos en la tensión entre distintas dinámicas sociales, particularmente entre el peso de los linajes y el de los funcionarios, pues mientras que los primeros tenían una base propia de poder político, los segundos dependían directamente del emperador. En términos de urbanismo, podemos conocer cómo el surgimiento del Imperio modificó la función de los viejos asentamientos, a la vez que fundó nuevos donde estableció los centros regionales de poder, cuestión que nos permite acercarnos al sistema y la jerarquía de estas "redes de ciudades".

Pasando ahora al Cercano Oriente, contamos con otros artículos donde también se aborda el urbanismo como un fenómeno regional formado por centros de distinto tamaño y función. Uno de ellos está dedicado a la Mesopotamia, "Lamministrazione dei centri minori della regione di Umma nel periodo protodinástico", redactado por Armando Bramanti, quien desde el enfoque de la geografía histórica intenta reconstruir la jerarquía de asentamientos de la región de Umma, permitiéndonos comprender las características del paisaje económico-administrativo impuesto por las ciudades sumerias en las áreas rurales. Otro artículo, en cambio, se ocupa de Egipto, "Trayectorias de urbanización en el valle y delta del Nilo en el IV milenio a.C.: Hieracómpolis y Tell el-Farkha en perspectiva comparada", cuya autoría pertenece a Marcelo Campagno, Marcin Czarnowicz y M. Belén Daizo. En este caso, se abordan las primeras experiencias urbanas, no sólo del Alto Egipto, espacio considerado como el epicentro de la urbanización y el origen del Estado en el Nilo, sino también del Bajo Egipto, particularmente del delta oriental, donde los 
hallazgos de las últimas décadas parecen indicar la existencia de un temprano foco de urbanización alternativo con sus propios patrones de desarrollo $\mathrm{y}$ funcionamiento.

Concentrándonos en el espacio estudiado en nuestro proyecto, Siria-Palestina, comenzamos señalando dos trabajos que también abordan la temática desde una escala regional. Por un lado, Leandro Constanze Lima, en su artículo "La expansión Uruk y el proceso de urbanización: una perspectiva desde la Alta Mesopotamia”, no sólo sintetiza las distintas teorías sobre la urbanización de esta región, sino que revisa la evidencia arqueológica de sitios ubicados en el Éufrates Medio y Alto, así como también en el Triángulo del Khabur, para concluir que, para explicar el surgimiento de las primeras ciudades, los vínculos interregionales son tan importantes como las condiciones locales. Ezequiel Cismondi, por su parte, en el artículo "Consideraciones sobre el rol del pastoralismo móvil en el proceso de urbanización en el sur del Levante durante el Bronce Antiguo", plantea que dicho proceso implicaba no solamente la emergencia de las primeras ciudades sino también la modificación de las formas de vida de las poblaciones móviles, las cuales comienzan a vincularse de manera sistemática con las poblaciones urbanas, ya sea en términos cordiales, por medio del intercambio o de la alianza, o en términos violentos, por medio de la guerra y de la resistencia.

Continuando con el primer urbanismo de Siria-Palestina, contamos con el artículo de Nimrod Getzov y Ianir Milevki, "Ciudades y luces a principios de la Edad del Bronce Antiguo. Sobre las primeras velas de aceite en el Levante meridional", quienes, a partir de un cuenco cerámico particular, hallado en cantidades significativas en distintos sitios del Bronce Antiguo sud-levantino, proponen que quizás pudo servir para alumbrar las salas interiores durante las largas jornadas de trabajo, lo que nos remite a la dependencia del artesanado especializado en el nuevo espacio de las ciudades. Sobre la crisis y el colapso de estas primeras urbes, Katherine Kifer, en su artículo "La destrucción de Jericó a fines de la Edad del Bronce Antiguo III: un análisis preliminar de diferentes interpretaciones", sugiere que, a pesar de las tendencias comunes hacia la desurbanización -vinculadas con una mayor orientación hacia el pastoralismo- que se constatan en todo el Levante meridional a partir de mediados del tercer milenio a.C., Jericó habría logrado persistir como un centro importante, sólo para caer después producto de la agresión de poblaciones móviles, tanto de las inmediaciones como quizás foráneas.

Por último, pasando a la Edad del Bronce Medio, del Bronce Tardío y la Edad del Hierro, disponemos de dos artículos, uno de Leticia Rovira y otro de Alejandro Mizzoni. El primero, titulado "El reino de Mari: ciudades, aldeas e identidades durante el período Paleobabilónico (2000-1600 a.C.)", vuelve a retomar el enfoque regional, combinando arqueología y filología. En su caso, logra identificar que la población de la ciudad capital es clasificada por las autoridades según su profesión o como jefes de unidades de trabajo, mientras que, en otras ciudades y aldeas, predomina una identificación clánica o étnica, contraste que nos revela la centralidad de la división del trabajo, característica propia del urbanismo, como ya hemos señalado. El segundo, "Aglomeración y dispersión poblacional en Siria entre el Bronce Tardío y el Hierro II", adopta una perspectiva de larga 
duración, partiendo de un contexto urbano, pasando por su crisis y llegando a su regeneración, pero concentrando las particularidades de este proceso en dos regiones distintas de Siria antigua. Entre las causas del fenómeno se sostiene el peso de la guerra, que obliga a las poblaciones dispersas a reunirse tras murallas. Pero más relevante aún resultan las consecuencias de estas aglomeraciones, que dan lugar a nuevas comunidades políticas, varias de las cuales se esfuerzan por vincularse, a través de la ocupación y/o reconstrucción de determinadas áreas y edificios, con centros prestigiosos del pasado.

En resumen, los distintos artículos que forman parte de este dossier analizan el fenómeno del urbanismo desde una amplia escala, considerando en sus análisis la relación entre las ciudades y otro tipo de asentamientos, incluso las poblaciones móviles. A su vez, tratan de establecer los criterios que ordenaban estos centros de manera jerárquica, así como las tensiones que los atravesaban, produciendo en ocasiones sus crisis. En lo que respecta al espacio interior de las ciudades, destacan el carácter heterogéneo de sus habitantes, donde la división del trabajo y las profesiones adquieren mayor relevancia, al menos desde la óptica de las autoridades, o bien donde grupos de distinto origen étnico ocupaban partes específicas del asentamiento, diferenciándose claramente de sus vecinos, como en el caso de Teotihuacan. ¿Acaso el carácter cosmopolita también sirva para definirlos como ciudades, a diferencia, por ejemplo, de las aldeas, que tenderían a ser más homogéneas en términos identitarios? ${ }^{11}$

Esta última pregunta, dejada para el final de manera adrede, sirve como ilustración de que el diálogo y el debate, tanto entre los integrantes de un proyecto -que en nuestro caso nuclea estudiantes y graduados con distinto grado de formación- así como con reconocidos investigadores de otras instituciones y países, es un elemento indispensable para seguir avanzando en la compresión de las distintas problemáticas históricas. Esperamos que esta publicación estimule la crítica y el planteo de nuevos interrogantes y que sirva para dar a conocer avances y resultados de investigación financiados por instituciones públicas de nuestro país.

\section{Agradecimientos}

Ante todo, quiero agradecer a la Secretaría de Investigación de la Facultad de Filosofía y Letras de la UBA, por habernos permitido llevar adelante este proyecto. Es importante destacar que algunos de los integrantes articulan sus investigaciones con otras dos iniciativas, ambas dirigidas por el Dr. Marcelo Campagno: PICT-Raíces 2015-2943 "Relaciones entre Egipto y Palestina a fines del IV milenio a.C.: hacia la construcción de un modelo multidisciplinario de interpretación", y UBACYT 20020160100059BA “Formas de conflicto en las sociedades del Mediterráneo antiguo". En mi caso particular, agradezco también

11 Véase también el artículo de Campagno, Czarnowicz y Daizo en este volumen. Tanto la definición de ciudad propuesta por Yoffee y Terrenato como por nosotros mismos más arriba, remite al carácter diverso de dichos asentamientos, es decir, de la alteridad al interior del espacio urbano. 
al Instituto Multidisciplinario de Historia y Ciencias Humanas, CONICET, por haberme otorgado una prórroga de mi beca postodoctoral, sin la cual hubiera sido difícil avanzar en la culminación de este proyecto, más aún en el contexto de pandemia que nos toca atravesar. En el curso de este trabajo también tuve la oportunidad de brindar tres seminarios, uno de posgrado en la Universidad Nacional de Salta (UNSa), y dos de grado en la UBA, lo que me permitió sintetizar parte de los avances y enriquecer mis reflexiones gracias al intercambio con los estudiantes. Para que esto último fuera posible conté con la amable invitación de Perla Rodríguez (UNSa), y del Departamento de Historia (UBA); a ellos dedico mis últimos agradecimientos. 
"Adams, R. McC. (1965). Land behind Baghdad. A History of Settlement on the Diyala Plains. Chicago: The University of Chicago Press.

» Adams, R. McC. (1966). The Evolution of Urban Society. Early Mesopotamia and Prehispanic Mexico. Chicago: Aldine.

»Algaze, G. (2008). La antigua Mesopotamia en los albores de la civilización. La evolución del paisaje urbano. Barcelona: Bellaterra.

»Algaze, G. (2018). Entropic Cities: The Paradox of Urbanism in Ancient Mesopotamia, en: Current Anthropology 59 (1): 23-54.

»Ashmore, W. (2008). Visions of the Cosmos: Ceremonial Landscapes and Civic Plans, en: David, B. y Thomas, J. (eds.), Handbook of Landscape Archaeology. Londres-Nueva York: Routledge, 167-175.

» Berry, B. J. L. y Pred, A. (1961). Central Place Studies: A Bibliography of Theory and Applications. Filadelfia: Regional Science Research Institute.

»Bietak, M., Czerny, E. y Forstner-Müller, I. (eds.) (2010). Cities and Urbanism in Ancient Egypt. Papers from a Workshop in November 2006 at the Austrian Academy of Sciences. Viena: Verlag der Österreichischen Akademie der Wissenschaften, 11-68.

»Biga, M. G. (2013). Defining the Chora of Ebla: A Textual Perspective, en: Matthiae, P. y Marchetti, N. (eds.), Ebla and Its Landscape. Early State Formation in the Ancient Near East. Walnut Creek: Left Coast Press, 259-267.

»Blanton, R. E. (1976). Anthropological Studies of Cities, en: Annual Review of Anthropology 5: 249-264.

» Carl, P., Kemp, B., Laurence, R., Coningham, R., Higham, C. y Cowgill, G. L. (2000). Were Cities Built as Images?, en: Cambridge Archaeological Journal 10 (2): 327-365.

»Carter, A., Heng, P., Stark, M., Chhay, R., y Evans, D. (2018). Urbanism and Residential Patterning in Angkor, en: Journal of Field Archaeology 43 (6): 492-506.

»Childe, V. G. (1950). The Urban Revolution, en: Town Planning Review 21 (1): 3-17.

»Christaller, W. (1980 [1933]). Die zentralen Orte in Süddeutschland. Eine ökonomisch-geographische Untersuchung über die Gesetzmäßigkeit der Verbreitung und Entwicklung der Siedlungen mit städtischer Funktion. Darmstadt: Wissenschaftliche Buchgesellschaft.

»Chueca Goitia, F. (1968). Breve historia del urbanismo. Madrid: Alianza.

"Clark, P. (ed.) (2013). The Oxford Handbook of Cities in World History. Oxford: Oxford University Press.

»Coe, M. D. (1961). Social Typology and Tropical Forest Civilizations, en: Comparative Studies in Society and History 4: 65-85.

»Cowgill, G. L. (2004). Origins and Development of Urbanism: Archaeological Perspectives, en: Annual Review of Anthropology 33: 525-549.

»Di Biagi, P. (dir.) (2014). Clásicos del urbanismo moderno. Bernal: Universidad Nacional de Quilmes. 
»Diego Espinel, A. (2002). Ciudades y urbanismo en el Egipto antiguo (CA. 300o1069 A.C.), en: Studia Historica. Historia Antigua 20: 15-38.

»Evans, D., Pottier, C., Fletcher, R., Hensley, S., Tapley, I., Milne, A. y Barbetti, M. (2007). A Comprehensive Archaeological Map of the World's Largest Preindustrial Settlement Complex at Angkor, Cambodia, en: Proceedings of the National Academy of Sciences 104: 14277-14282.

» Farhat, G. (ed.) (2020). Landscapes of Preindustrial Urbanism. Washington D. C.: Dumbarton Oaks Research Library and Collection.

» Finet, A. (1982). Lorsque la royauté descendit du ceil. Les foullies belges du Tell Kannâs sur l'Euphrate en Syrie. Mariemont: Musée royal de Mariemont.

»Flannery, K. V. (1998). The Ground Plan of Archaic States, en: Feinman, G. M. y Marcus, J. (eds.), Archaic States. Santa Fe: School of American Research Press, 15-57.

» Fox, R. G. (1977). Urban Anthropology. Cities in Their Cultural Settings. Englewood Cliffs, NJ: Prentice-Hall.

» Frangipane, M. y Manzanilla, L. R. (2018). Dossier Rethinking Urbanization and Its Living Landscapes from the Inspiring Perspective of a Great “Maestro", en: Origini 42 (2). Roma: Gangemi Editori International.

»Fustel de Coulanges (2009 [1866]). La cité antique: Étude sur le culte, le droit, les institutions de la Grèce et de Rome. Cambridge: Cambridge University Press.

» Garfinkel, Y. y Ben-Shlomo, D. (2009). Sha'ar Hagolan 2. The Rise of Urban Concepts in the Ancient Near East. Jerusalén: The Hebrew University of Jerusalem.

»Gloria Estúa, F. (2003). Los complejos piramidales y el urbanismo en Egipto, en: Wiesheu, W. (ed.), Evocaciones de Asia y África. México D. F.: Instituto Nacional de Antropología e Historia, 25-42.

» Harmanşah, Ö. (2013). Cities and the Shaping of Memory in the Ancient Near East. Cambridge: Cambridge University Press.

» Hirth, K. G. (1978). Interregional Trade and the Formation of Prehistoric Gateway Communities, en: American Antiquity 43 (1): 35-45.

» Hodder, I. y Orton, C. (1990 [1976]). Análisis espacial en Arqueología. Barcelona: Crítica.

» Jacobs, J. (1975 [1969]). La economía de las ciudades. Barcelona: Península.

»Johnson, G. A. (1972). Local Exchange and Early State Development in Southwestern Iran. Anthropological Papers 51. Ann Arbor: Museum of Anthropology, University of Michigan.

»Khazanov, A. M. (1984 [1983]). Nomads and the Outside World, Second Edition. Madison, Wisconsin: The University of Wisconsin Press.

» Liverani, M. (2014 [2013]). Imaginar Babel. Dos siglos de estudios sobre la ciudad oriental antigua. Barcelona: Bellaterra.

»López Barja de Quiroga, P. L. y Lomas Salmonte, F. J. (2004). Historia de Roma. Madrid: Akal.

» Lull, V.y Micó, R. (2007). Arqueología del origen del Estado. Las teorías. Barcelona: Bellaterra.

" Mandich, M. J. (2019). Ancient City, Universal Growth? Exploring Urban Expansion and Economic Development on Rome's Eastern Periphery, en: Frontiers of Digital Humanities 6: 18, doi: 10.3389/fdigh.2019.00018. 
" Marcus, J. y Sabloff, J. A. (eds.) (2008). The Ancient City: New Perspectives on Urbanism in the Old and New Worlds. Santa Fe: School for Advanced Research Press.

" Marfoe, L. (1979). The Integrative Transformation: Patterns of Sociopolitical Organization in Southern Syria, en: Bulletin of the American Schools of Oriental Research 234: 1-42.

"Milevski, I. (2010). Centros urbanos y periferias en la Edad del Bronce Antiguo sud-levantina, en: Rivista degli Studi Orientali 83 (1-4): 163-187.

"Moeller, N. (2016). The Archaeology of Urbanism in Ancient Egypt. From the Predynastic Period to the End of the Middle Kingdom. Nueva York: Cambridge University Press.

»Mumford, L. (2012 [1961]). La ciudad en la historia. Sus orígenes, transformaciones y perspectivas. Logroño: Pepitas de Calabaza.

»Osborne, J. F. (2014). Settlement Planning and Urban Symbology in SyroAnatolian Cities, en: Cambridge Archaeological Journal 24 (2): 195-214.

»Osterhammel, J. (2015 [2013]). La transformación del mundo. Una historia global del siglo XIX. Barcelona: Crítica.

»Parlebas, J. (1983). La notion de niout (localité) dans le pensée égyptienne antique, en: Brüschweiler, F. (ed.), La ville dans le Proche Orient ancien. Actes du Colloque de Cartigny. Leuven: Peeters, 199-207.

»Redman, Ch. L. (1990 [1978]) Los orígenes de la civilización. Desde los primeros agricultores hasta la sociedad urbana en el Próximo Oriente. Barcelona: Crítica.

» Rosen, S. A. (2017). Revolutions in the Desert. The Rise of Mobile Pastoralism in the Negev and the Arid Zones of the Southern Levant. Nueva York-Londres: Routledge.

»Service, E. (1984 [1975]). Los orígenes del estado y de la civilización. El proceso de la evolución cultural. Madrid: Alianza Universidad.

» Silver, M. (2004). Modern Ancients, en: Rollinger, R. y Ulf, C. (eds.), Commerce and Monetary Systems in the Ancient World: Means of Transmission and Cultural Interaction. Múnich: Franz Steiner, 65-87.

»Sjoberg, G. (1960). The Preindustrial City. Past and Present. Glencoe: Free Press.

"Smith, M. L. (ed.) (2003). The Social Construction of Ancient Cities. Washington D. C.: Smithsonian Institute Press.

»Soja, E. W. (2008 [2000]). Postmetrópolis. Estudios críticos sobre las ciudades y las regiones. Madrid: Traficantes de sueños.

»Stone, E. (2005). Mesopotamian Cities and Countryside, en: Snell, D. C. (ed.), A Companion to the Ancient Near East. Malden-Oxford-Carlton: Blackwell Publishing, 141-154.

»Storey, G. R. (ed.) (2006). Urbanism in the Preindustrial World. Cross-Cultural Approaches. Tuscaloosa: The University of Alabama Press.

»Thomas, A. R. (2012). Urbanization before Cities: Lessons for Social Theory from the Evolution of Cities, en: Journal of World-Systems Research 18 (2): 211-235.

» Tilley, C. (1997). A Phenomenology of Landscape: Places, Paths and Monuments. Oxford: Berg.

» Trigger, B. G. (1972). Determinants of Urban Growth in Pre-Industrial Societies, en: Ucko, P. J., Tringham, R. y Dimbleby, G. W. (eds.), Man, Settlement, and 
Urbanism. Cambridge: Schenkman, 575-599.

" van de Mieroop, M. (1997). The Ancient Mesopotamian City. Oxford: Clarendon Press.

" van Dike, R. M. (2008). Memory, Place, and the Memorization of Landscape, en: David, B. y Thomas, J. (eds.), Handbook of Landscape Archaeology. LondresNueva York: Routledge, 277-284.

»Verderame, L. (2010). La imagen de la ciudad en la literatura sumeria, en: Rivista degli Studi Orientali 83 (1-4): 23-48.

"Verhoeven, M. (2006). Megasites in the Jordanian Pre-Pottery Neolithic B: Evidence of Proto-Urbanism?, en: Banning, E. B. y Chazan, M. (eds.), Domesticating Space. Construction, Community, and Cosmology in the Late Prehistoric Near East. Studies in Early Near Eastern Production, Subsistence, and Environment 6. Berlín: Ex Oriente, 75-79.

»Weber, M. (1944 [1922]). Economía y Sociedad. Esbozo de sociología comprensiva. México-Buenos Aires: Fondo de Cultura Económica.

"Wheatley, P. (1971). The Pivot of the Four Quarters. A Preliminary Enquiry into the Origins and Character of the Ancient Chinese City. Chicago: Aldine.

»Wickham, Ch. (2013 [2009]). El legado de Roma. Una historia de Europa de 400 a 1000. Barcelona: Pasado y Presente.

»Wilson, J. A. (1960). Egypt through the New Kingdom: Civilization without Cities, en: Kraeling, C. H. y Adams, R. McC. (eds.), City Invincible. A Symposium on Urbanization and Cultural Development in the Ancient Near East Held at the Oriental Institute of the University of Chicago, December 4-7, 1958. Chicago: The University of Chicago, 124-136.

»Wright, H. T. y Johnson, G. A. (1975). Population, Exchange, and Early State Formation in Southwestern Iran, en: American Anthropologist 77 (2): 267-289.

»Yoffee, N. (ed.) (2015). Early Cities in Comparative Perspective, 4000 BCE - 1200 CE. The Cambridge World History III. Cambridge: Cambridge University Press.

"Yoffee, N. y Terrenato, N. (2015). Introduction: A History of the Study of Early Cities, en: Yoffee, N. (ed.), Early Cities in Comparative Perspective, 4000 BCE 1200 CE. The Cambridge World History III. Cambridge: Cambridge University Press, 1-24. 
